\section{¿Cómo aprenden los estudiantes de medicina en la transición hacia el ciclo clínico? Estudio cualitativo de las percepciones de estudiantes y docentes acerca del aprendizaje inicial de la clínica}

\author{
MARCELA BITRAN ${ }^{1, \mathrm{a}}$, DENISSE ZÚÑIGA ${ }^{1, \mathrm{~b}}$, ISABEL LEIVA², \\ MARIBEL CALDERÓN ${ }^{3, c}$, ALEMKA TOMICIC ${ }^{3, d}$, \\ OSLANDO PADILLA ${ }^{4, e}$, ARNOLDO RIQUELME ${ }^{1,5}$
}

\section{Perceptions of students and teachers about clinical medicine learning}

Background: The transition to the clinical courses represents a major challenge for medical students who are expected to become experiential learners, able to integrate theory and practice in the context of patient care. There are questions about how students face this challenge. Aim: To understand and compare the perceptions of students and clinical tutors on how medical students learn during the transition to the clinical levels of the curriculum. Material and Methods: We performed eight focus group discussions with 54 students enrolled in years three to seven and we interviewed eight clinical tutors. Both students' focus group discussions and tutors' interviews were audio recorded, transcribed and analyzed according to the Grounded Theory. Results: Nine main themes emerged from the analysis of students' opinions and six from the tutors' views. The following themes were common to both students and educators: educational activities, actors, clinical settings, learning strategies, transition markers and tutor's role. Educators emphasized the importance of curricular courses' design and students, that of emotions, adaptation and self-care strategies, and threats to learning. Conclusions: There is a common core of students' and clinical tutors' perceptions about the relevance of practical activities, social interactions and context in the development of students' learning and adaptation strategies during the transition to the clinical levels of the curriculum. These results are related to social and cultural theories of learning. Thus we propose a model for early clinical learning that might help to stimulate the reflection of students and medical educators regarding clinical learning and contribute to the development of interventions that improve the clinical learning and teaching practices.

(Rev Med Chile 2014; 142: 723-731)

Key words: Models, educational; Students, medical; Teaching.
${ }^{1}$ Centro de Educación Médica, Escuela de Medicina, Pontificia Universidad Católica de Chile. ${ }^{2}$ Departamento de Enfermedades Respiratorias, Escuela de

Medicina, Pontificia Universidad Católica de Chile.

${ }^{3}$ Escuela de Psicología, Pontificia Universidad Católica de Chile. ${ }^{4}$ Departamento de Salud Pública, Escuela de Medicina, Pontificia Universidad Católica de Chile.

${ }^{5}$ Departamento de

Gastroenterología, Escuela de Medicina, Pontificia Universidad

Católica de Chile.

aBioquímica, PhD en

Farmacología.

bPsicóloga, Magister en Psicología Educacional.

'Licenciada en Educación, estudiante de doctorado en Psicología Educacional. 'Psicóloga, PhD en Psicología Clínica.

eEstadístico.

Proyecto financiado por el Fondo Nacional de Desarrollo Científico y Tecnológico, (Fondecyt) proyectos\#1120534 (M.B.) y \#1120652 (A.R.)

Recibido el 26 de diciembre de 2013, aceptado el 14 de junio de 2014.

Correspondencia a: Marcela Bitran Fono: 23543811 mbitran@med.puc.cl
L a docencia clínica es de vital importancia para la adquisición de dos competencias médicas fundamentales: las habilidades para realizar la entrevista clínica y el examen físico, y para comunicarse eficazmente con el paciente $y$ su familia ${ }^{1}$. El currículo de pregrado de la Escuela de Medicina de la Pontificia Universidad Católica de Chile, así como el de diversas universidades del país, contempla un período intermedio entre el ciclo básico y el internado, en que el estudiante 
es introducido gradualmente a la práctica clínica ${ }^{2}$. En este período, las metodologías de enseñanza propias de las ciencias básicas dan paso a nuevas y variadas formas de enseñar en escenarios de diversa complejidad y autenticidad, y la evaluación se hace más completa, integrando aspectos cognitivos, relacionales y actitudinales ${ }^{2}$.

Durante esta transición -la primera de muchas en la formación del médico- se pone a prueba la capacidad de adaptación de los estudiantes, y se siembran las bases de la identidad profesional ${ }^{3-5}$. Según Teunissen y Westerman (2011), parte de la dificultad propia de este período tiene relación con las expectativas de los estudiantes. Éstos ingresan a los ambientes clínicos esperando ser educados y se encuentran con escenarios en que se requiere un aprendizaje experiencial y autodirigido ${ }^{3}$. De hecho, la progresión del estudiante desde las ciencias básicas a la clínica y luego, su transición de estudiante a médico involucra un creciente uso del aprendizaje experiencial ${ }^{6-8}$. Así lo demuestra un estudio longitudinal realizado en la Escuela de Medicina de la Pontificia Universidad Católica de Chile, que indica que los estudiantes de medicina cambian sus modos típicos de aprender a medida que avanzan en los estudios, transformándose -de aprendices asimiladores (que observan y reflexionan) al comienzo de la carrera- en aprendices convergentes (que observan y hacen) al finalizar ésta ${ }^{9}$.

El aprendizaje clínico, como otros aprendizajes en contextos de trabajo, se ha intentado explicar mediante teorías socio-culturales, tales como el aprendizaje situado y las comunidades de práctica ${ }^{8,10}$, que relevan los aspectos sociales por sobre los individuales y meramente cognitivos. No obstante, hasta el momento existe escaso sustento empírico para esta propuesta. Un estudio cualitativo que aborda este tema apoya el rol de 'lo social' en el aprendizaje clínico de los residentes ${ }^{11}$. Sin embargo, se sabe poco de lo que ocurre en el pregrado, lo que es particularmente necesario en Chile y otros países donde los estudiantes ingresan a las universidades muy jóvenes, provenientes de una formación escolar fuertemente marcada por un aprendizaje pasivo ${ }^{12}$.

¿Cómo se transforman los estudiantes de medicina en aprendices experienciales? ¿Qué estrategias usan para hacer sentido de la experiencia clínica $y$ aprender en contextos de cuidado al paciente?

El objetivo de este estudio es conocer y comparar las percepciones de estudiantes y docentes respecto de cómo aprenden la clínica los estudiantes de medicina en la transición del ciclo básico al clínico.

\section{Materiales y Métodos}

\section{Diseño de la investigación}

El diseño de este estudio se basó en el uso de la metodología cualitativa, que permite una orientación hacia el descubrimiento del objeto de investigación, e implica la simultaneidad entre la selección de la muestra, la recolección de datos y el análisis. Utilizamos el enfoque de Teoría Fundada ${ }^{13,14}$, que reconoce la naturaleza social del fenómeno de estudio, y una perspectiva constructivista, que asigna al investigador un rol participativo en la construcción de la teoría resultante $\mathrm{e}^{15,16}$.

Este estudio, de carácter cualitativo y descriptivo, pretende construir -en base al relato de los participantes- un modelo que explique cómo los estudiantes de medicina aprenden la clínica.

Los resultados informados en este artículo corresponden a la fase de indagación cualitativa de un proyecto Fondecyt destinado a desarrollar un instrumento para identificar las aproximaciones al aprendizaje de la clínica durante la transición al ciclo clínico.

Este estudio fue aprobado por el comité de Ética de la Escuela de Medicina de la Pontificia Universidad Católica de Chile y por Fondecyt.

\section{Participantes}

\section{Estudiantes}

Participaron 54 estudiantes de medicina que cursaban entre $3^{\text {er }}$ y $6^{\circ}$ año durante 2012 (Tabla 1 ), seleccionados mediante un muestreo intencionado considerando criterios de sexo y año de estudios. Los estudiantes fueron invitados a participar en una reunión grupal (grupo focal) vía correo electrónico $y$, quienes aceptaron, firmaron un consentimiento informado antes de su realización.

\section{Docentes}

Participaron ocho docentes clínicos (Tabla 1). Los criterios de inclusión fueron: ser jefe de curso o tutor clínico de $3^{\text {er }}$ a $6^{\circ}$ año de medicina, con más de cuatro años de experiencia docente, de ambos sexos. Los docentes fueron entrevistados 
Tabla 1. Participantes del estudio

\begin{tabular}{|ccc|}
\hline \multicolumn{2}{|c|}{ Estudiantes } & \\
Curso & n & Hombres/mujeres \\
$3^{\circ}$ & 15 & $9 / 6$ \\
$4^{\circ}$ & 8 & $4 / 4$ \\
$5^{\circ}$ & 14 & $6 / 8$ \\
\hline $6^{\circ}$ & 17 & $10 / 7$ \\
\hline Total & 54 & $29 / 25$ \\
\hline
\end{tabular}

\begin{tabular}{|c|c|c|c|}
\hline \multicolumn{2}{|c|}{ Tutores clínicos } & \multirow[b]{2}{*}{$\begin{array}{l}\text { Años de } \\
\text { docencia }\end{array}$} & \multirow[b]{2}{*}{ Curso } \\
\hline $\begin{array}{l}\text { Entrevistado } \\
\text { (E) }\end{array}$ & Sexo & & \\
\hline E1 & M & 4 & $4^{\circ}$ \\
\hline E2 & M & 21 & $5^{\circ}$ \\
\hline E3 & M & 10 & $5^{\circ}$ \\
\hline E4 & M & 18 & $3^{\circ}$ \\
\hline E5 & M & 21 & $4^{\circ}$ \\
\hline E6 & $\mathrm{F}$ & 5 & $4^{\circ}$ \\
\hline E7 & M & 7 & $1^{\circ}$ a $7^{\circ}$ \\
\hline E8 & M & 11 & $4^{\circ}$ \\
\hline
\end{tabular}

M: masculino, F: femenino.

individualmente, previa firma de consentimiento informado. La participación fue voluntaria.

\section{Grupos focales}

Se realizaron ocho grupos focales con estudiantes de $3^{\text {er }}$ a $6^{\circ}$ año (dos grupos focales por nivel) entre agosto y noviembre de 2012. La reunión (60 a $90 \mathrm{~min}$ ) -conducida por dos investigadores del equipo- se inició con preguntas abiertas, destinadas a indagar las percepciones de los estudiantes acerca de cómo aprenden a integrar la teoría con la práctica clínica y a comunicarse con los pacientes.

\section{Entrevistas}

Se usó un formato semiestructurado, con preguntas abiertas destinadas a explorar las percepciones de los docentes de cómo aprenden sus estudiantes a integrar teoría y práctica, y a comunicarse con los pacientes. Las entrevistas (60 a $90 \mathrm{~min}$ ) se realizaron entre abril y julio de 2012 y fueron conducidas por los miembros del equipo de investigación. Los registros de audio se transcribieron.

\section{Análisis de los datos}

El análisis se realizó en paralelo con la recolección de datos, con el fin de mejorar los guiones de entrevistas y grupos focales; la recolección de datos se prolongó hasta alcanzar la saturación de los temas, o sea, hasta cuando la recolección de información no reportara información nueva. Los registros de audio transcritos se analizaron según la Teoría Fundada, que propone enfrentar el análisis con una mente abierta, sin categorías 'a priori'. Este enfoque permite descubrir, desarrollar y verificar provisionalmente teorías emergentes 'enraizadas' en los datos, que expliquen los fenómenos en estudio, a partir de un proceso de recolección y análisis de datos sistemático y en relación recíproca. La construcción del modelo presentado en este estudio incluyó las etapas descriptiva e interpretativa. Los datos se agruparon en categorías temáticas en base a la codificación abierta de los conceptos aludidos por los participantes, y a la codificación axial, que propone relaciones entre las categorías de conceptos de la codificación abierta ${ }^{13,14}$. La generación de códigos y categorías se orientó por el criterio de descripción densa, es decir, procurando comprender e interpretar contextualizadamente las respuestas ${ }^{16}$. Con el propósito de asegurar la calidad de los resultados, se empleó la estrategia de triangulación, que implica que las categorías propuestas por un investigador fueron validadas a través del acuerdo intersubjetivo entre todos los investigadores ${ }^{17}$. La codificación de los datos se apoyó con el uso del programa ATLAS.TI versión WIN 7.1@

\section{Resultados}

\section{Temas emergentes}

Frente a la pregunta: ¿Cómo aprenden la clínica los estudiantes de medicina?, estudiantes y docentes participaron activamente proporcionando abundante material para la investigación. Los conceptos construidos desde la codificación abierta de los grupos focales de estudiantes se organizaron en nueve temas principales y los de las entrevistas de docentes, en siete. Seis temas comunes estuvieron presentes en ambos relatos (Tabla 2).

En la Tabla 3 se presentan viñetas ilustrativas de cada tema, ordenados alfabéticamente. Bajo el título: 'actividades educativas' se organizan 
Tabla 2. Temas emergentes

\begin{tabular}{|llcc|}
\hline \multicolumn{2}{|l}{ Temas } & Estudiantes & Tutores clínicos \\
\hline 1. Actividades educacionales & $\bullet$ & $\bullet$ \\
\hline 2. Actores & $\bullet$ & $\bullet$ \\
\hline 3. Amenazas al aprendizaje & $\bullet$ & $\bullet$ \\
4. Campos clínicos & $\bullet$ & \\
\hline 5. Emociones & $\bullet$ & $\bullet$ \\
6. Estrategias de adaptación y autocuidado & $\bullet$ & $\bullet$ \\
7. Estrategias de aprendizaje & $\bullet$ & $\bullet$ \\
8. Estructura de cursos & & $\bullet$ \\
9. Marcadores de aprendizaje & $\bullet$ & $\bullet$ \\
10. Rol del tutor & & $\bullet$ \\
\hline
\end{tabular}

Tabla 3. Citas representativas de estudiantes y tutores clínicos

\begin{tabular}{|c|c|c|}
\hline Temas & Estudiantes & Tutores clínicos \\
\hline $\begin{array}{l}\text { Actividades } \\
\text { educacionales }\end{array}$ & $\begin{array}{l}\text { "Cuando llegaba la tutora, elegíamos uno de los } \\
\text { casos [...], lo discutíamos con la tutora [...] veíamos } \\
\text { la fisiopatología, [...], el examen físico [...], des- } \\
\text { pués íbamos a ver a ese paciente todo el grupo; } \\
\text { esa era la dinámica". (GF1 tercero; P52) }\end{array}$ & $\begin{array}{l}\text { "Tienen una reunión de razonamiento clínico que } \\
\text { está calendarizada del principio de la rotación en } \\
\text { que cada alumno tiene un día asignado y está } \\
\text { con un docente asignado. Nos rotamos entre los } \\
\text { docentes que estamos en la posta; todo el grupo } \\
\text { de cuarto, que son } 20 \text { alumnos, que están con } \\
\text { un docente y liderado por uno de ellos. La sesión } \\
\text { la prepara el alumno que implica que recibe la } \\
\text { instrucción de escoger un caso interesante para } \\
\text { discutir y que la idea es que lo presente completo; } \\
\text { ellos nunca han presentado un caso clínico de un } \\
\text { paciente, entonces, para ellos es un desafío desde } \\
\text { la elección del caso...". (E6; P44) }\end{array}$ \\
\hline Actores & $\begin{array}{l}\text { "En general, para ese tipo de tareas, uno tiene casi } \\
\text { siempre la figura de un becado (residente) que, } \\
\text { en general, son bien cercanos con uno y tratan de } \\
\text { dejar las cosas prácticas bien claras al inicio, y las } \\
\text { dudas, [...].se resuelven a través de los becados". } \\
\text { (GF1 sexto; P34) }\end{array}$ & $\begin{array}{l}\text { "Con quiénes resuelven las dudas habitualmente } \\
\text { no es con el tutor, sino que prefieren resolverlo con } \\
\text { alguien que sea más cercano, que es el interno o el } \\
\text { residente o becado de la sala". (E8; P47) }\end{array}$ \\
\hline $\begin{array}{l}\text { Amenazas al } \\
\text { aprendizaje }\end{array}$ & $\begin{array}{l}\text { "En las mañanas se pasaba una visita [...]; en la } \\
\text { tarde, muchas veces volvíamos a pasar la misma } \\
\text { visita. Entonces, era bien contraproducente". (GF2 } \\
\text { sexto; P55) }\end{array}$ & \\
\hline Campos clínicos & $\begin{array}{l}\text { "Dermatología era en 'San Joaquín' y Traumatolo- } \\
\text { gía, [...] en Sotero del Rio, o en (el hospital) UC. Se } \\
\text { mueve en esos tres lugares [...] lo que uno hace, } \\
\text { va dependiendo también del lugar que a uno le } \\
\text { toque". (GF2 cuarto; P31) }\end{array}$ & $\begin{array}{l}\text { "En la semana, tienen un día, al menos, que van a } \\
\text { policlínico [...] y, habitualmente, son dos o tres ve- } \\
\text { ces a la semana (que) entran a pabellón". (E3; P51) }\end{array}$ \\
\hline Emociones & $\begin{array}{l}\text { "En tercero, [...] era como el primer enfrentamien- } \\
\text { to: había más miedo, más susto. Como que uno no } \\
\text { cachaba mucho pero, de tanto ver pacientes, como } \\
\text { que uno se suelta y -al final- yo por lo menos, } \\
\text { como ya estoy más tranquilo". (GF1 quinto; P370) }\end{array}$ & \\
\hline
\end{tabular}


¿Cómo aprenden los estudiantes de medicina en la transición hacia el ciclo clínico? - M. Bitran et al

\begin{abstract}
Estrategias de adaptacion y autocuidado

Estrategias de aprendizaje

"Uno se adapta a un montón de situaciones como de ambientes también o sea el ambiente de cirugía es distinto al de acá y como que todos los servicios de salud funcionan un poco distinto y las habilidades sociales: saber cómo comportarse, como saber desarrollarse. Y uno pasa harto solo ya no es como el mundo que pasa en el grupo piola en las clases, si no que en el fondo te evalúan más personalmente" (GF1 sexto; P166)

"Puede que un compañero sepa más que uno y le puede enseñar lo que ya aprendió. Yo he practicado mucho todas las entrevistas, he practicado con compañeros y [...] saben todos los síntomas que tenemos que tener y me los describen bien, entonces es una buena forma de practicar las enfermedades estándar". (GF1 tercero; P163)
\end{abstract}

Estructura de curso

Marcadores de transición
Rol del tutor
"Estudian solos, pero les gusta juntarse en grupo para repasar o revisar preguntas, [...] ejercitan las mismas pruebas [...]. Preguntan en grupo, se preparan para exámenes en grupo, se preparan para ECOE en grupo". (E5; P82)

"Yo dirijo un curso que se llama XX [...] Ocupa prácticamente ochenta y cinco por ciento del tiempo que los alumnos tienen destinado al período académico de cuarto año. Es un curso integrado que tiene actividades prácticas en las mañanas y que tiene actividades teóricas con clases en la tarde". (E5; P2)

"El alumno de cuarto no tiene esa urgencia de saber de un día para otro, o sea, de saber en el momento, y el interno sí. Por ejemplo, yo le digo a los alumnos de cuarto: no sabemos esto, repasemos de aquí a mañana. iYa! dicen ellos [...] en cambio, el interno tiene como una especie de urgencia por saber". (E1; P47) en tercero uno lo pregunta todo y ahora uno ya sabe qué es lo que tiene que preguntar, qué es lo que tiene que hacer, como acotar las cosas". (GF1 sexto; P114)

"Algo que encontré muy rescatable de nuestra tutora, que intentaba incomodar lo menos posible a nuestra paciente y ien qué se notaba aquello? la discusión de la historia clínica jamás era al lado de la cama (del paciente), menos cuando era algo sensible [...]. Esa modalidad, yo la encontré muy, muy ética, práctica y educativa". (GF1 tercero; P114)
"Yo trataba de demostrarles con ejemplos más o menos simples, de la importancia de ir agregándole datos a la anamnesis, para eso traté de usar esquemas un poco diferentes de lo tradicional y engancharlos". (E2; P192)

GF: grupo focal, P: párrafo, E: entrevista.

descripciones de diversas actividades curriculares, por ejemplo, la visita al paciente y la presentación de un caso clínico.

El tema 'actores' alude a diversas personas relacionadas con la práctica clínica (p. ej. compañeros, residentes y pacientes) y su importancia en el proceso de aprendizaje.

'Amenazas al aprendizaje', presente solo en el relato de los estudiantes, es un tema que aglutina situaciones diversas, concebidas por ellos como pérdida de tiempo u obstáculos al aprendizaje.
'Campos clínicos' aparece en el relato de docentes y estudiantes como los contextos concretos en que ocurren los aprendizajes.

'Emociones', tema exclusivo de los estudiantes, incluye descripciones de emociones experimentadas durante las actividades prácticas, muchas de ellas asociadas a contextos de inexperiencia o error.

'Estrategias de adaptación y autocuidado', tema que los estudiantes identificaron adicionalmente a las de aprendizaje, alude a diversas 
conductas orientadas a responder a las nuevas exigencias y también a mantener el bienestar integral. 'Estrategias de aprendizaje' es un tema central del relato de estudiantes y docentes e incluye actividades individuales o grupales orientadas al aprendizaje práctico y/o teórico.

La 'estructura de los cursos', tema exclusivo de docentes, detalla la estructura de los cursos integrados de $4^{\circ}$ y $5^{\circ}$ año.

'Marcadores de transición', concepto acuñado por el equipo de investigación,se refiere a características y/o conductas de los estudiantes que permitirían reconocer a quienes han realizado con éxito la transición a la clínica.

Los tutores clínicos emergen como agentes cruciales para el aprendizaje, tanto en la percepción de los estudiantes como de los propios docentes. Por ello, los códigos referentes a ellos se organizaron en una categoría nueva denominada 'rol del tutor'.

\section{Modelo general de cómo aprenden los estudiantes}

La Figura 1 ilustra un modelo que organiza los conceptos expresados por docentes y estudiantes acerca de cómo aprenden los estudiantes de me- dicina durante la transición al ciclo clínico. En el centro se representa la relación recíproca entre las actividades curriculares y las estrategias de los estudiantes para enfrentarlas -incluyendo tanto estrategias orientadas al aprendizaje como a la adaptación y al autocuidado. Este proceso recursivo ocurre en un contexto emocional y social en el que también participan múltiples actores ( $\mathrm{p}$. ej. tutor, paciente, compañeros, residente). Estos actores interactúan con el estudiante y también entre ellos. Las actividades e interacciones tienen lugar en diversos campos clínicos que, con sus dinámicas asistenciales propias, constituyen los escenarios físicos en que ocurre el aprendizaje clínico.

\section{Discusión}

El objetivo de este estudio fue conocer y contrastar las percepciones de estudiantes y docentes acerca de cómo aprenden los estudiantes de medicina durante los años de transición a la clínica. Pese a poseer distintos énfasis, las visiones de docentes y estudiantes mostraron una coherencia básica expresada en la presencia de temas comunes. Basados en ellos, proponemos un modelo del

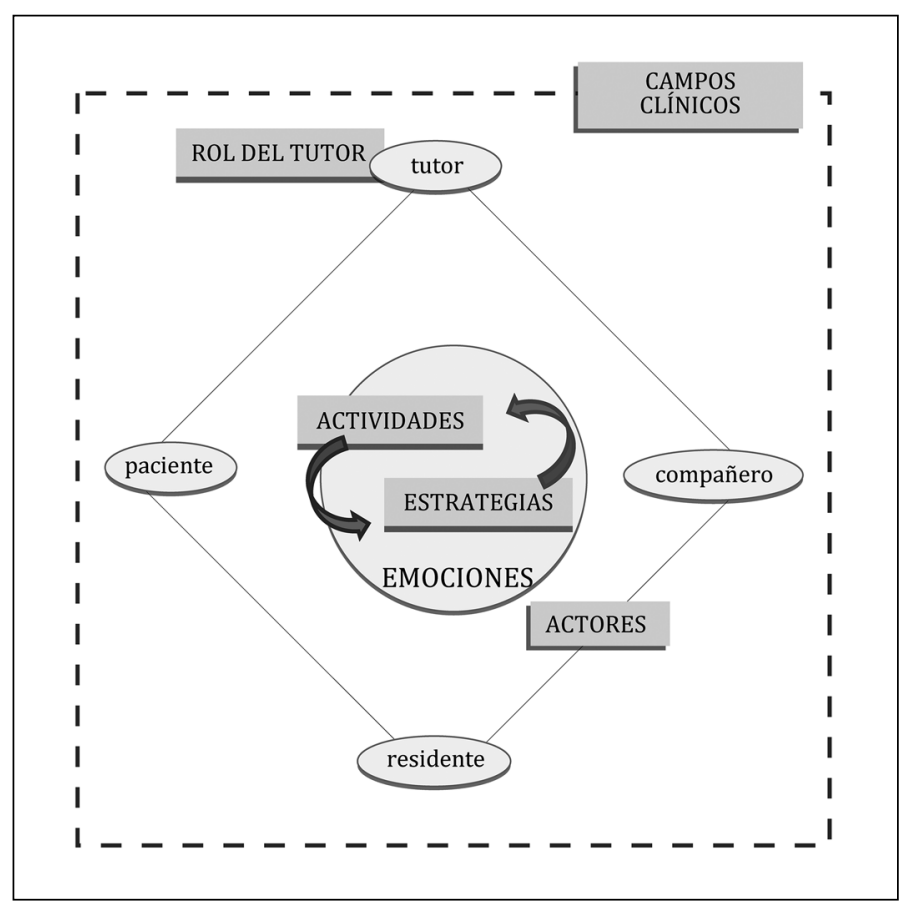

Figura 1. Modelo del aprendizaje temprano de la clínica. 
aprendizaje clínico cuyo centro es la adaptación del estudiante a las demandas impuestas por las actividades prácticas. Esta adaptación se manifiesta en estrategias de aprendizaje y autocuidado que el estudiante desarrolla en interacción con sus compañeros, residentes, tutores clínicos y pacientes. Dichas estrategias son influidas por el ambiente emocional y por las posibilidades y restricciones propias de los campos clínicos. Una adaptación exitosa a esta nueva y compleja forma de aprender se manifestaría en una serie de características que hemos denominado 'marcadores de transición'.

El modelo propuesto es consistente con las teorías socio-culturales del aprendizaje que asignan un papel relevante a la dimensión social y situada de éste ${ }^{12,18,19}$. Según estas teorías, el aprendizaje clínico involucraría al estudiante en su capacidad de relacionarse con múltiples actores y actuar diferenciadamente según el contexto ${ }^{6,18,19}$. Esto resuena con el relato de nuestros estudiantes quienes refieren que es necesario establecer redes para satisfacer distintas necesidades de aprendizaje: compañeros para recibir y dar feedback y apoyo, residentes para resolver dudas, pacientes para mantenerse motivados por aprender, y tutores clínicos como modelos a imitar (o evitar), y para resolver situaciones complejas.

Nuestros hallazgos son consistentes con los de Teunissen y colaboradores que revelan que la participación en actividades prácticas y la interacción con pares son elementos centrales en el aprendizaje clínico de los residentes ${ }^{20,21}$.

Wenger va más allá al proponer que el aprendizaje en contextos de trabajo, el caso del aprendizaje clínico, no es construido por los estudiantes en interacción con otros, sino por la comunidad de práctica de la que el estudiante forma parte ${ }^{19}$. El término 'comunidad de práctica', acuñado inicialmente por Lave y Wenger (1991),se refiere a un grupo de personas con diversidad de experiencias que comparte un interés o profesión, y que aprenden unos de otros ${ }^{18}$. Para calificar como miembros,los individuos deben ser legítimos, periféricos y participantes ${ }^{19}$. Este sería el caso del estudiante de medicina en la comunidad de práctica clínica pues su presencia en las actividades clínicas está establecida curricularmente (es legítima), su rol es periférico (la responsabilidad clínica recae en el tutor o el médico tratante) y participativo, pues es a través de su participación en las actividades de la unidad que él aprende ${ }^{6}$. Más aún, hay evidencias que las actividades continuas de los estudiantes y su involucramiento en comunidades de práctica clínica influyen en sus aproximaciones y estrategias de aprendizaje $e^{22,23}$.

Un elemento diferenciador del discurso de los docentes y estudiantes que participaron en este estudio es que los primeros atribuyen mayor importancia a las metodologías de enseñanza y a la estructura de los cursos. De hecho, cuando se les preguntó cómo aprenden sus estudiantes, los docentes hablaron extensamente sobre cómo ellos enseñan. Este sesgo podría originarse en la premisa -propia de las teorías cognitivas- que el estudiante se limita a aprender lo que se le enseña ${ }^{8}$, sin participar en la construcción del conocimiento. Este punto merece explorarse en el futuro, ya que las concepciones del docente acerca de cómo ocurre el aprendizaje influencian sus prácticas pedagógicas.

Otros elementos diferenciadores del discurso de estudiantes y docentes son las emociones y las estrategias de adaptación y autocuidado, temas mencionados exclusivamente por los estudiantes. Éstos relatan que las emociones que experimentan en el contexto de las actividades clínicas afectan su disposición y aproximaciones para aprender. Además, refieren que estudiar es una dimensión inseparable de los demás aspectos de sus vidas y que éstas se modifican como una manera de 'sobrevivir' a las nuevas demandas académicas.

Los hallazgos reportados aquí son consistentes con evidencias que indican que el 'cómo nos sentimos' afecta la manera en que percibimos e interpretamos la información, y también influye en cómo se aprenden y transfieren las habilidades clínicas ${ }^{24}$. Además, se ha demostrado que las emociones no solo afectan cómo aprendemos sino también nuestra motivación por aprender ${ }^{25,26}$. Por ejemplo, el orgullo y alegría activan el aprendizaje, mientras que emociones como la vergüenza, el miedo o el aburrimiento, reducen la motivación por aprender ${ }^{26}$. En la misma línea, van der Zwet y colaboradores, basándose en un estudio cualitativo con estudiantes de $5^{\circ}$ año de medicina, concluyen que para aprender la práctica médica y desarrollar su identidad profesional, los estudiantes necesitan un 'espacio de desarrollo' en que se entremezclan las emociones con las interacciones personales y profesionales en el contexto de trabajo ${ }^{27}$.

Llama la atención que el tema 'emociones' y 'adaptación' estuvo virtualmente ausente del discurso de los docentes entrevistados. Aunque 
estos refieren que algunos estudiantes pasan por períodos de estrés y ansiedad, no relacionan directamente las emociones cotidianas de sus estudiantes con el aprendizaje. Es posible que esto se deba a que los docentes se formaron en un paradigma educacional que enfatiza los aspectos cognitivos por sobre los emocionales y personales.

El modelo aquí propuesto da cuenta de la diversidad y riqueza de las experiencias de aprendizaje de los estudiantes durante la transición a la clínica, y de la mirada de sus docentes en una escuela de medicina chilena. A poco de ingresar a los cursos clínicos, los estudiantes desarrollan estrategias para responder a las exigencias de las nuevas actividades educacionales que involucran competencias cognitivas, prácticas y actitudinales. Esta relación estrategia-actividad se nutre del contacto social con diversos actores -entre los que destaca el tutor y los pares- y se modifica según los contextos específicos en que ocurre el aprendizaje clínico. El éxito del estudiante para hacer frente a las exigencias académicas de esta etapa parece relacionarse con su capacidad de autorregulación y adaptación; procesos que no aparecen en el relato de sus tutores clínicos.

Reconocemos que una de las limitaciones del estudio fue realizarlo en una sola escuela de medicina, por lo que no es posible establecer cuán aplicable es a otros contextos. En la actualidad estamos realizando un panel Delphi con estudiantes y docentes de diversas escuelas de medicina del país para confeccionar un cuestionario de las estrategias de aprendizaje inicial de la clínica. Los resultados de este panel nos permitirán evaluar el grado de validez nacional de los conceptos y relaciones derivados del presente estudio.

En conclusión, este estudio contribuye con evidencias empíricas a la idea que el aprendizaje inicial de la clínica es un proceso social y situado, que no es adecuadamente explicado por las teorías cognitivas. La transición a la clínica marca la 'iniciación' del estudiante en el aprendizaje experiencial, que lo compromete íntegramente (física, racional, emocional y socialmente) como miembro de una comunidad cuyo sentido se realiza en la atención al paciente.

Esperamos que este estudio estimule la reflexión de estudiantes y educadores médicos y genere bases para diseñar intervenciones que potencien el aprendizaje y la docencia en la transición a la clínica.
Agradecimientos: Agradecemos a todos los estudiantes y docentes que participaron en este estudio.

\section{Referencias}

1. Harden $\mathrm{H}$ and Laidlaw J. In Essential skills for a medical teacher: an introduction to teaching and learning in medicine, Elsevier, Churchill Livingstone; 2012.

2. Sánchez I, Riquelme A, Moreno R, Mena B, Dagnino J, Grebe G. Revitalizing medical education: the school of medicine at the Pontificia Universidad Católica de Chile. Clin Teacher 2008; 5: 57-61.

3. Teunissen P, Westerman M. Opportunity or threat: Ambiguity in the consequences of transitions in medical education. Med Educ 2011; 45: 51-9.

4. Prince KJAH, van der Wiel MWJ, Scherpbier AJJA, van der Vleuten CPM, Boshuizen HPA. A qualitative analysis of the transition from theory to practice in undergraduate training in a PBL-medical school. Adv Health Sci Educ 2000; 5: 105-16.

5. Helmich E, Bolhuis S, Dornan T, Laan R, Koopmans R. Entering medical practice for the very first time: emotional talk, meaning and identity development. Medical Education 2012; 46: 1074-87.

6. Dornan T, Boshuizen H, King N, Scherpbier A. Experience-based learning: A model linking the processes and outcomes of medical students' workplace learning. Med Educ 2007; 41: 84-91.

7. Nicola B, Corrigan O, Allard J, Archer J, Barnes R, Bleakley A, et al. The transition from medical student to junior doctor: today's experiences of Tomorrow's Doctors, Medical Education 2010; 44 (5): 449-58.

8. Yardley S, Teunissen P, Dornan T. AMEE guides: Theories in Medical Education. Experiential Learning AMEE guide No. 63 Medical Teacher 2012; 34: e10215.

9. Bitran M, Zúñiga D, Pedrals N, Padilla O, Mena B. Medical students' change in learning styles during the course of undergraduate program: from 'thinking and learning' to 'thinking and doing'. Canadian Medical Education Journal 2012 3(2): e86-e97.

10. Durning S, Artino A. Situativity theory: A perspective on how participants and the environment can act. Med Teach 2011; 33: 188-99.

11. Bernabeo E, Holtman M, Ginsburg S, Rosenbaum J, Holboe E. Lost in transition: the experience and impact of frequent changes in the in-patient learning environment. Acad Med 2011; 86: 591-8.

12. Martinic S. Uso del tiempo e interacciones profesores- 
alumnos en la sala de clases. En La Evaluación Docente en Chile. Centro de Medición Mide UC. 2011.

13. Charmaz K. Constructing Grounded Theory: A Practical Guide Through Qualitative Analysis. Thousand Oaks, Calif: Sage; 2006.

14. Straus A, Corbin J. Basics of Qualitative Research: Grounded Theory Procedures and Techniques. Thousand Oaks, Calif: Sage; 1990.

15. Watling CJ, Lingard L. Grounded theory in medical education research: AMEE guide no. 70. Med Teach 2012; 34: 850-61.

16. Ponterotto J. Brief note on the origins, evolution, and meaning of the qualitative research concept "Thick Description". The Qualitative Report 2006; 11: 538-49.

17. O'Cathain A, Murphy E, Nicholl J. Three techniques for integrating data in mixed methods studies. BMJ 2010; 341: c4587.

18. Lave J, Wenger E. Situated learning: Legitimate peripheral participation. Cambridge: Cambridge University Press; 1991.

19. Wenger E. Communities of practice: Learning, meaning and identity. New York, NY: Cambridge University Press; 1998.

20. Teunissen P, Scheele F, Scherpbier A, van der Vleuten C, Boor K, van Luijk S, et al. How residents learn: qualitative evidence for the pivotal role of clinical activities. Med Educ 2007; 41: 763-70.

21. Teunissen P, Boor K, Scherpbier A, van der Vleuten C, van Diemen-Steenvorde J, van Luijk S, et al. Attending doctors' perspectives on how residents learn. Med Educ 2007; 41 (11): 1050-8.

22. Lave J, Chaiklin S. Understanding practice: Perspectives on activity and context. Cambridge: University of Cambridge Press. 1993.

23. Al Kadri HMF, Al-Moamary MS, Elzubair M, Magzoub ME, AlMutairi A, Roberts C, et al. Exploring factors affecting undergraduate medical students' study strategies in the clinical years: a qualitative study. Adv in Health Sci Educ 2011; 16: 553-67.

24. McConnell M, Eva K. The role of emotion in the learning and transfer of clinical skills and knowledge. Acad Med 2012; 87 (10): 1316-22.

25. Artino A, Holmboe E, Durning S. Can achievement emotions be used to better understand motivation, learning, and performance in medical education? Medical Teacher 2012; 34: 240-4.

26. Artino A, Holmboe E, Durning SJ. Control-value theory: Using achievement emotions to improve understanding of motivation, learning, and performance in medical education: AMEE Guide no. 64. (Theories in Medical Education). Dundee: AMEE; 2012.

27. van der Zwet J, Zwietering $P$, Teunissen $P$, van der Vleuten C, Scherpbier A. Workplace learning from a socio-cultural perspective: creating developmental space during the general practice clerkship. Adv Health Sci Educ Theory Pract 2011; 16 (3): 359-73. 